\title{
HECKE RINGS OF CONGRUENCE SUBGROUPS
}

\author{
BY NELO D. ALLAN
}

Communicated by M. Gerstenhaber, December 27, 1971

Let $\boldsymbol{k}$ be a $\mathfrak{p}$-adic field and let $\hat{\boldsymbol{G}}$ be a reductive group defined over $\boldsymbol{k}$. Let $\boldsymbol{G}$ be a semigroup in $\hat{\boldsymbol{G}}$, i.e. a multiplicative subset with the same unity as $\hat{G}$ We shall assume that there exists an open compact subgroup $\Delta$ of $\hat{\boldsymbol{G}}$ which is contained in $\boldsymbol{G}$. Let $\mathscr{R}(\boldsymbol{G}, \Delta)$ be the free $\boldsymbol{Z}$-module generated by the double cosets of $G$ modulo $\Delta$, with a product defined as in [3, Lemma $6]$. We have an associative ring with unity which we shall call the Hecke Ring of $G$ with respect to $\Delta$. Let $\Delta_{0}$ be a normal subgroup of $\Delta$ satisfying our conditions $\mathrm{H}-1$ and $\mathrm{H}-2$ of $\$ 1$. Our purpose is to find generators and relations for $\mathscr{R}\left(G, \Delta_{0}\right)=\mathscr{R}$. There exists a finitely generated polynomial ring $Z[G]$ which together with the group ring $Z\left[\Delta / \Delta_{0}\right]$ generates $\mathscr{R}$; moreover $\mathscr{R}$ is a $Z\left[\Delta / \Delta_{0}\right]$-bimodule having $Z[D]$ as basis. Our hypothesis $\mathrm{H}-1$ and $\mathrm{H}-2$ are verified for the principal congruence subgroups of most of the classical groups considered in [2].

We thank Mr. J. Shalika for the helpful discussions during the preparation of this work and also for pointing out some hopes that this might bring in solving Harish-Chandra conjecture on the finite dimensionality of the irreducible continuous representations of these rings.

1. General results. Let $\boldsymbol{T}$ be a connected $\boldsymbol{k}$-closed subgroup of $\boldsymbol{G}$ consisting only of semisimple elements, and $\boldsymbol{N}^{+}$and $\boldsymbol{N}^{-}$be maximal $\boldsymbol{k}$ closed unipotent subgroups normalized by $T$. We set $N^{+}=N^{+} \cap \Delta$, and $U^{-}=N^{-} \cap \Delta$. We shall now state our first condition:

Condition H-1. There exists a finitely generated semigroup $D$ in $T$ such that $G=\Delta D \Delta$ (disjoint union), and for all $d \in D$ we have $d U^{+} d^{-1}$ $\subset U^{+}$and $d^{-1} U^{-} d \subset U^{-}$.

We turn now to our second condition. We let $\Delta_{0}$ be a normal subgroup of $\Delta$ and we set $U_{0}^{+}=U^{+} \cap \Delta_{0}$ and $U_{0}^{-}=U^{-} \cap \Delta_{0}$. We shall assume that $T \cdot N^{+} \cap \Delta_{0}=\left(T \cap \Delta_{0}\right) \cdot U_{0}^{+}$.

Condition H-2. There exists a semigroup $D$ in $T$ such that $\Delta_{0}=U_{0}^{+} V U_{0}^{-}$ for a certain subgroup $V$ of $\Delta_{0}$ normalized by $D$, and for all $d$ in $D$ we have $d U_{0}^{+} d^{-1} \subset U_{0}^{+}$and $d^{-1} U_{0}^{-} d \subset U_{0}^{-}$.

Let us denote by $\overline{1}$ the unity of $\mathscr{R}$ and by $\bar{g}$ the double coset $\Delta_{0} g \Delta_{0}$. We shall denote the product in $\mathscr{R}$ by $*$.

TheOREM 1. Condition H-2 implies that $D=\Delta_{0} D \Delta_{0}$ is a semigroup in $\hat{\boldsymbol{G}}$ and $\mathscr{R}\left(\hat{D,} \Delta_{0}\right) \simeq Z[D]$.

AMS 1969 subject classifications. Primary 2220; Secondary 2265, 4256, 2070.

Key words and phrases. Hecke rings, algebraic groups, locally compact groups, convolution algebras. 
Proof. Our condition implies that for all $d_{1}, d_{2} \in D$, we have $\Delta_{0} d_{1} \Delta_{0} d_{2} \Delta_{0}$ $=\Delta_{0} d_{1} d_{2} \Delta_{0}$, or $\bar{d}_{1} * \bar{d}_{2}=m \cdot \bar{d}_{1} d_{2}$, with $m \in Z$, and it remains to prove that $m=1$. From H-2 we can write

$$
\Delta_{0} d \Delta_{0}=\bigcup\left\{\Delta_{0} d u_{j} \mid j=1, \ldots, \omega(d), u_{j} \in \boldsymbol{U}_{0}^{-}\right\} .
$$

Set $v_{j}=d u_{j} d^{-1} \in N^{-}$. If $\Delta_{0} d u_{j}=\Delta_{0} d_{1}$ for some $d_{1}$ in $\boldsymbol{D}$, then we have $\bar{v}_{j}=\overline{1}$ and we may replace $d_{1}$ by $d$. This is equivalent to the existence of $v \in \Delta_{0}$ such that $v d=d u_{j}$. Now we set $\Delta_{0} d_{i} \Delta_{0}=\bigcup \Delta_{0} d u_{j}^{(i)}, i=1,2$, and we recall that $m$ is the number of pairs $(i, j)$ such that

$$
\Delta_{0} d_{1} d_{2}=\Delta_{0} d_{1} u_{i}^{(1)} d_{2} u_{j}^{(2)} \text {. }
$$

We have $v d_{1} d_{2}=d_{1} d_{2} \tilde{u}_{i}^{(1)} u_{j}^{(2)}=d_{1} u_{i}^{(1)} v_{j}^{(2)} d_{2}$, for some $\tilde{u}_{i}^{(1)}$ in $\Delta_{0}$, and this implies $\bar{v}_{j}^{(2)}=1$ and consequently we have $\bar{v}_{i}^{(1)}=\overline{1}$. Therefore $m=1$. Q.E.D.

THEOREM 2. The conditions $\mathrm{H}-1$ and $\mathrm{H}-2$ with the same $D$ imply the finite generation as a ring of $\mathscr{R}$. Moreover $\mathscr{R}$ is a $Z\left[\Delta / \Delta_{0}\right]$-bimodule having $\boldsymbol{Z}[\boldsymbol{D}]$ as a basis.

Proof. We let $\left\{d_{1}, \ldots, d_{r}\right\}$ be a set of generators for $D$. Let $\left\{\alpha_{1}, \ldots, \alpha_{h}\right\}$ be a complete set of representatives for $\Delta$ modulo $\Delta_{0}$. Normality of $\Delta_{0}$ implies that $\overline{\alpha_{i} d \alpha_{j}}=\bar{\alpha}_{i} * d * \bar{\alpha}_{j}$. Also we have $\bar{\alpha}_{i} \bar{\alpha}_{j}=\bar{\alpha}_{i} * \bar{\alpha}_{j}$ and for any $d, d^{\prime} \in D, \overline{d d^{\prime}}=d * \bar{d}^{\prime}$. Now H-1 implies that for any $g \in \boldsymbol{G}$ there exist $\alpha_{i}, \alpha_{j} \in \Delta$ and $d \in D$ such that $\bar{g}=\overline{\alpha_{i} d \alpha_{j}}$ and also that for any $h$ in $\Delta$ and any $1 \leqq i, j \leqq r, d_{i} * \overline{h d_{j}}=\bar{d}_{i} * \bar{h} * \bar{d}_{j}=\bar{d}_{i} h * d_{j}$ is a linear combination with coefficients in $\boldsymbol{Z}$ of the elements $\overline{\alpha_{i} d \alpha_{j}}$. Therefore the number of generators of $\mathscr{R}$ is $r \cdot h_{0}$, where $h_{0}$ is the minimal number of generators of $\Delta / \Delta_{0}$. Q.E.D.

2. Relations. We observe that Theorem 2 gives us some relations among the generators of $\mathscr{R}$. Let us introduce some notation; for fixed $d \in \boldsymbol{D}$, we shall let $L(d)$ (resp. $\boldsymbol{R}(d))$ be the set of $\left\{\bar{\alpha} \mid \alpha \in \Delta, \overline{\alpha d}=\overline{d \alpha^{\prime}}\right.$, for some $\alpha^{\prime} \in \Delta$ (resp. $\left.\left.\overline{d \alpha}=\overline{\alpha^{\prime} d}\right)\right\}$. $L(d)$ and $R(d)$ are subgroups of $\bar{\Delta}$. We denote by $R^{\prime}(d)$ and $\boldsymbol{L}^{\prime}(d)$ the respective subgroups of $\boldsymbol{R}(d)$ and $\boldsymbol{L}(d)$ consisting of those elements $\bar{\alpha}$ such that $\bar{\alpha}^{\prime}$ can be chosen as $\overline{1}$. It is easy to verify that $R^{\prime}(d)=\left\{\bar{\alpha} \mid \alpha \in d^{-1} U_{0}^{+} d \cap \Delta\right\}=\overline{d^{-1} \Delta_{0} d \cap \Delta}$ and $L^{\prime}(d)=\{\bar{\alpha} \mid \alpha$ lies in $\left.d U_{0}^{-} d^{-1} \cap \Delta\right\}=\overline{d \Delta_{0} d^{-1} \cap \Delta}$. We have the following straightforward lemmas:

LEMMA 1. $\bar{\alpha}_{i} * \bar{d} * \bar{\alpha}_{r}=\bar{\alpha}_{j} * \bar{d}^{\prime} * \bar{\alpha}_{s}$ if and only if $\bar{d}=\bar{d}^{\prime}, \bar{\alpha}_{j} \in \bar{\alpha}_{i} * L(d)$ and $\bar{\alpha}_{s} \in \boldsymbol{R}^{\prime}(d) * \bar{\psi} * \bar{\alpha}_{r}, \overline{\alpha_{i}^{-1} \alpha_{j}} * \bar{d}=d * \bar{\psi}$ for some $\psi \in \Delta$.

Lemma 2. Suppose that for all the generators $d$ of $D$ we have $d U_{0}^{-} d^{-1}$ $\subset \boldsymbol{U}^{-}$. If $d$ and $d^{\prime}$ are generators of $\boldsymbol{D}$ and if $g \in \Delta$, then

$$
d * \bar{g} d^{\prime}=\theta(d, g) * \bar{\alpha} * \bar{d}_{1} * \bar{\alpha}^{\prime},
$$


where $\alpha, \alpha^{\prime} \in \Delta$ and $d_{1} \in D$ are such that $\overline{d g d^{\prime}}=\overline{\alpha d_{1} \alpha^{\prime}}$, and $\theta(d, g)=m \cdot($ sum of all elements of $L^{\prime}(d) W$, where $W$ is the subgroup of $L^{\prime}(d)$ consisting of all $\bar{\alpha}$ such that $\bar{\alpha} * \overline{d g d^{\prime}}=\overline{d g d^{\prime}}$ ). $m$ is not greater than the order of the group

$$
\overline{\mathrm{g}} * \boldsymbol{L}^{\prime}\left(d^{\prime}\right) * \overline{\boldsymbol{g}}^{-1} \cap \boldsymbol{R}^{\prime}(d) \text {. }
$$

Finally, we would like to observe that $\mathscr{R}$ has an involution induced by $g \rightarrow g^{-1}$ in the case where $G$ is a group. If, moreover, there exists $\theta \in \Delta$ such that for all $d \in D, d^{-1}=\theta^{-1} d \theta$, then the mapping $\bar{\alpha} \rightarrow \overline{\theta \alpha \theta^{-1}}$ induces the isomorphisms $R^{\prime}(d) \simeq L^{\prime}(d)$ and $R(d) \simeq L(d)$.

EXAMPLES. Let $K$ be a division algebra central over $k, D$ be the ring of integers of $K, \mathfrak{p}$ its prime and $\pi$ a fixed generator of $\mathfrak{p}$. Given $a \in K$ we shall denote by ord $(a)$ the power of $\pi$ in $a$. We let $q$ be the number of elements in $\mathfrak{O} / \mathfrak{p}$. For any positive integer $m, \mathfrak{O} / \mathfrak{p}^{m}$ has $q^{m}$ elements. Let $S$ be a subring of $K$ and let $M_{n}(S)$ denote the ring of all $n$ by $n$ matrices with entries in $S$; if $g \in M_{n}(S)$ and $1 \leqq i, j \leqq n$, then $(g)_{i j}$ will denote the $(i, j)$-entry of $g$ and if we set $(g)_{i j}=g_{i j}$, we write $g=\left(g_{i j}\right)$; by $e_{i j}$ we denote the matrix having 1 as $(i, j)$-entry and zero otherwise, and $E_{n}$ or simply $E$ will denote the identity of $M_{n}(S) . G l_{n}(K)$ is the group of units of $M_{n}(K)$.

Case I. $\hat{G}=G l_{n}(K)$. We let $\boldsymbol{G}=\boldsymbol{G l}(\boldsymbol{K}), \boldsymbol{T}=\boldsymbol{T}_{n}=$ diagonal matrices in $G, N^{+}$(resp. $N^{-}$) the group of all unipotent upper (resp. lower) triangular matrices in $\boldsymbol{G}, \Delta=\boldsymbol{G}_{\mathfrak{D}}=\boldsymbol{G} l_{n}(\boldsymbol{D})$. Let $\boldsymbol{D}_{n}=\left\{d \in \boldsymbol{T} \mid d=\operatorname{diag}\left[\pi^{r_{1}}, \ldots, \pi^{r_{n}}\right]\right.$, $\left.r_{1} \geqq r_{2} \geqq \cdots \geqq r_{n}\right\}$. It is clear that $D_{n}$ satisfies H-1. For any $r \geqq 1$ we set $\Delta_{0}$ $=\Delta_{r}=\left\{g \in \Delta \mid g=1 \bmod \mathfrak{p}^{r}\right\}=$ the $r$ th congruence subgroup of $\Delta$. We have $T \cdot N^{+} \cap \Delta_{r}=\left(T \cap \Delta_{r}\right) \cdot U_{0}^{+}$. We let $V=T \cap \Delta_{r}$ and $V^{\prime}=T \cap \Delta$. Condition H-2 will follow from the following lemma:

$$
\text { LEMMA 3. } \Delta_{r}=U_{0}^{+} \boldsymbol{V} U_{0}^{-}=U_{0}^{-} V U_{0}^{+} \text {. }
$$

Proof. Let $g \in \Delta_{r}$. As $V$ normalizes both $U_{0}^{+}$and $U_{0}^{-}$we may assume that all diagonal entries of $g$ are 1 . If we consider $g^{\prime}=\left(E-g_{i n} e_{i n}\right) g$, $i \neq n$, then $E-g_{i n} e_{i n} \in U_{0}^{+}$and $\left(g^{\prime}\right)_{i n}=0$. These operations will reduce to zero the nondiagonal entries of the last column of $g$. Now it suffices to transpose the resulting matrix, repeat the operation and apply induction. Q.E.D.

REMARK. Let $d \in D$ be such that $r_{n} \geqq 0$. For any $\bar{v} \in L^{\prime}(d)$ we can choose a representative such that $d^{-1} v d=u=\left(u_{i j}\right) \in U^{-}$where $u_{i j}=0$, for $i<j, u_{i i}=1$ for all $i$, and $u_{i j}=a_{i j} \pi^{r}$ with $\operatorname{ord}\left(a_{i j}\right)<r_{j}-r_{i}$. Hence $\omega(d)=q^{m}, m=\sum_{i<j}\left(r_{j}-r_{i}\right)$. Also $d^{-1}=\theta d \theta, \theta=e_{1 n}+\cdots+e_{n 1}$ and for the generators of $D, d^{-1} \Delta_{r} d$ and $d \Delta_{r} d^{-1}$ are contained in $\Delta_{r-1}$, because $r_{1}=1$.

Finally we would like to remark that in the case $n=2$ and $K=k$ we have $m(Z(u) w, d)=1$ if $u$ is a unit, and equal to $q$, otherwise, where 


$$
d=\left(\begin{array}{ll}
\pi & 0 \\
0 & 1
\end{array}\right), \quad w=\left(\begin{array}{ll}
0 & 1 \\
1 & 0
\end{array}\right), \quad Z(u)=\left(\begin{array}{ll}
1 & 0 \\
u & 1
\end{array}\right) .
$$

Also if $u$ is a unit, $d * \overline{Z(u) w d}=\overline{d Z(u) w d}$. This well determines the multiplication in $\mathscr{R}$.

The case where $G=S l_{n}(k)$, and $D, \Delta, \Delta_{r}, V$ being the respective intersection of the corresponding groups with $\boldsymbol{S l}_{n}(\boldsymbol{k})$, is covered by our Theorem 2.

Case II. Unitary groups. Let $\boldsymbol{K}$ denote either $\boldsymbol{k}$, or a quadratic extension of $\boldsymbol{k}$, or else a quaternion division algebra over $\boldsymbol{k}$. Let $\rho$ denote respectively, the identity, the nontrivial automorphism of $\boldsymbol{K}$ over $\boldsymbol{k}$, and an involution of $\boldsymbol{K}$. Clearly $\rho$ can always be extended to an involution of $M_{n}(K)$. Let $h \geqq 0$ and let $n=2 p+b$; we subdivide every matrix $g \in M_{n}(K)$ into 9 blocks $g=\left(g_{i j}\right), i, j=1,2,3$, in such way that $g_{11}, g_{33} \in M_{p}(K)$ and $g_{22} \in M_{b}(K)$. Let $\mathfrak{D}$ be the ring of integers of $K$, and fix an $H \in M_{n}(D)$, such that $H^{\rho}=\gamma H, \gamma= \pm 1, H=\left(h_{i j}\right), h_{13}=\gamma h_{31}=\theta=e_{1 \rho}+\cdots+e_{\rho 1}$, $h_{22}=V$ and $h_{i j}=0$ otherwise, where $V$ corresponds to an anisotropic form, if $b \neq 0$. We let $G$ be the connected component of the group $\left\{g \in \boldsymbol{G l}_{n}(\boldsymbol{K}) \mid g^{\rho} \mathrm{Hg}=\mu(g) H\right.$, where $\mu$ is the multiplier $\}$, and we let $\boldsymbol{G}_{0}$ be the correspondent group of $V$. We let

$$
\boldsymbol{T}=\left\{h \in \boldsymbol{G} \mid h=\operatorname{diag}\left[z, h_{0}, \mu\left(h_{0}\right) \theta\left(z^{\rho}\right)^{-1} \theta\right], h_{0} \in \boldsymbol{G}_{0} \text { and } z \in \boldsymbol{T}_{p}\right\},
$$

and we denote by $N^{+}, N^{-}, \Delta$ and $\Delta_{r}$ the intersection of the corresponding group in $\boldsymbol{G l}_{n}(\boldsymbol{K})$ with $\boldsymbol{G}$. We let $\boldsymbol{V}=\boldsymbol{T} \cap \Delta_{r}$ which clearly normalizes $\boldsymbol{U}_{0}^{+}$and $\boldsymbol{U}_{0}^{-}$.

LEMMA 4. $\Delta_{r}=U_{0}^{+} V U_{0}^{-}$.

Proof. Let $g=\left(g_{i j}\right) \in \Delta_{r}$. We can apply Lemma 3 to $g_{33}$ and we can write $g_{33}=n_{1} h_{1} u_{1}$. If we denote by $n=\operatorname{diag}\left[\theta\left(n_{1}^{\rho}\right)^{-1} \theta, E, n_{1}\right], h=$ $\operatorname{diag}\left[\theta\left(h_{1}^{\rho}\right)^{-1} \theta, E, h_{1}\right]$ and $u=\operatorname{diag}\left[\theta\left(u_{1}^{\rho}\right)^{-1} \theta, E, u_{1}\right]$, then $n \in U_{0}^{+}, h \in V$, and $u \in U_{0}^{-}$and replacing $g$ by $h^{-1} n^{-1} g u^{-1}$ we may assume that $g_{33}=E$. We take now $g^{\prime}=\left(g_{i j}^{\prime}\right) \in U_{0}^{+}$with $g_{12}^{\prime}=\gamma \theta g_{23}^{\rho} V, g_{13}^{\prime}=\gamma \theta g_{13}^{\rho} \theta$ and $g_{23}^{\prime}=-g_{23}$ and $g^{\prime \prime}=\operatorname{diag}\left[E, h_{0}, E\right]$ in $V$, for a convenient $h_{0} \in G_{0}$; hence $g^{\prime \prime} g^{\prime} g \in U_{0}^{-}$. Q.E.D.

Now we consider $\Lambda$ as in $[2, \S 9], l=0, D=\left\{\pi^{r} \mid r \in \Lambda\right\}$ and $D^{\prime}$ $=\{d \in \Delta \mid \mu(d)=1\}$. We take $\hat{\boldsymbol{G}}=\boldsymbol{G}$ and $\boldsymbol{G}^{\prime}=\{g \in \boldsymbol{G} \mid \mu(g)=1\}$ and consider their respective subgroups $\Delta, \Delta^{\prime}, V, V^{\prime}$, etc. It can be easily checked that in all the cases discussed in $[2, \S 9]$, our Theorems 1 and 2 remain valid for $\left(G^{\prime}, \Delta^{\prime}\right)$ and for $(G, \Delta)$ with the exception of the case $(O) n=2 p$. For $G^{\prime}$ we also have the extra assumptions of Lemma 2 and we also have a $\theta=\left(\theta_{i j}\right) ; \theta_{13}=\gamma \theta_{31}=\theta, \theta_{22}=E, \theta_{i j}=0$ otherwise, such that $d^{-1}$ $=\theta d \theta, \pi^{\rho}=\gamma \pi$.

Closing this note we shall make two remarks: 
REMARK. For the adjoint representation of a Chevalley type group we have condition $\mathrm{H}-1$ by [1].

Remark. Let $\mathscr{H}$ be a Hilbert space and let $\mathscr{B}(\mathscr{H})$ be the algebra of all bounded operators on $\mathscr{H}$. Suppose that there exists a finite group $\boldsymbol{G}$ of unitary operators and a finite set of commuting operators $D_{1}, \ldots, D_{r}$ all in $\mathscr{B}(\mathscr{H})$ such that all $D_{i}$ 's are not necessarily normal. Let $\mathscr{B}$ be the weak closure of the algebra generated by 1 and all the $D_{i}$. If we assume that every $A \in \mathscr{B}(\mathscr{H})$ can be written as a finite sum of $g_{i} B_{i j} g_{j}, g_{i}, g_{j} \in G$ and $B_{i j} \in \mathscr{B}$, does this necessarily imply that the dimension of $\mathscr{H}$ is finite? The positive answer of this question together with our Theorem 2 will imply Harish-Chandra's conjecture in these cases.

\section{REFERENCES}

1. N. Iwahori and H. Matsumoto, On some Bruhat decomposition and the structure of the Hecke rings of p-adic Chevalley groups, Inst. Hautes Études Sci. Publ. Math. No. 25 (1965), 5-48. MR 32 \# 2486.

2. I. Satake, Theory of spherical functions on reductive algebraic groups over p-adic fields, Inst. Hautes Études Sci. Publ. Math. No. 18 (1963), 5-69. MR 33 \#4059.

3. T. Tamagawa, On the ל-function of a division algebra, Ann. of Math. (2) 77 (1963), 387-405. MR 26 \# 2468.

Universidad Nacional de Colombia, Bogota, Colombia, South America

Department of Mathematics, University of Wisconsin at Parkside, Racine, WISCONSIN 53403 